\title{
Internato de medicina geral e familiar em época pandémica: o que (com)promete?
}

Ana Margarida Dias Marques, ${ }^{1}$ Ana Lúcia Silva Pereira ${ }^{1}$

\section{RESUMO}

O plano de formação em medicina geral e familiar corresponde a um processo bem definido de formação médica contínua que, a partir de março de 2020, se viu balançado na sua estrutura, em detrimento da mobilização dos médicos internos para áreas de atuação não planeadas, mas de extrema importância no combate à pandemia por COVID-19. Neste contexto, houve um desvio do percurso formativo que, embora sentido de forma desigual pelos diferentes anos do internato médico, se revelou transversal, existindo uma potencial quebra do fio condutor da aprendizagem da medicina geral e familiar. Não obstante, a pandemia veio constituir uma força motriz para o médico interno desenvolver novas competências e em áreas até então por explorar, das quais se destacam a aquisição de noções de trabalho em equipa e competências comunicacionais, bem como o aperfeiçoamento das técnicas de consulta telefónica. Também o binómio interno-orientador se tornou mais coeso, pela aprendizagem conjunta em tempo real, e registou-se um enfoque da atividade clínica em detrimento das tarefas curriculares. Sugerem-se estudos que se continuem a debruçar sobre o grau de motivação dos médicos internos de medicina geral e familiar e sobre a forma como as suas novas competências poderão constituir elementos de valorização individual.

Palavras-chave: COVID-19; Pandemia; Internato médico; Medicina geral e familiar.

\section{INTRODUÇÃO}

$\square$ m 1974 surgiu na Europa, em Leeuwenhorst, uma definição de médico de família (MF) num documento denominado O CLÍNICO GERAL NA EUROPA, baseada na definição profissional produzida por um grupo de trabalho do Royal College of General Practitioners, em 1972. ${ }^{1-2}$ Nessa altura a medicina geral e familiar (Clínica Geral/Medicina Familiar) estava na sua infância enquanto disciplina, especialmente no que diz respeito à sua base de ensino e investigação. As duas definições que se seguiram, a da WONCA de 1991 e a de Olesen de 2000, pareciam ter muitas raízes na definição de Leeuwenhorst; no entanto, a definição daWONCA pareceu ter-se tornado mais relevante para os diferentes sistemas de saúde ao incorporar alguns descritores da disciplina. ${ }^{3}$

Após algumas atualizações da sua definição e, mais recentemente, segundo aWONCA 2011, a medicina ge-

1. Médica Interna de Medicina Geral e Familiar. USF Vida+, ACeS Cávado II - Gerês/Cabreira. Vila Verde, Portugal. ral e familiar (MGF) apresenta-se como uma disciplina e como uma especialidade, uma vez que comporta uma componente académica e científica, dotada de conteúdos educacionais próprios, bem como se direciona para a prática dos cuidados de saúde primários (CSP), ${ }^{3}$ a pedra basilar do Serviço Nacional de Saúde.

Em Portugal, a criação da especialidade e a sua integração em Diário da República remonta ao ano de 1982 e, desde então, que o programa de formação específica de MGF tem vindo a sofrer reformulações e atualizações. Importa particularizar a publicação do Decreto-Lei n. ${ }^{\circ}$ 45/2009, de 13 de fevereiro, e da Portaria n. ${ }^{\circ} 300 / 2009$, de 24 de março, em que se propõe o programa de formação em MGF como um processo bem definido e devidamente estruturado de formação médica especializada, teórica e prática, tendo como objetivo central habilitar o médico de uma prática científica e tecnicamente diferenciada. ${ }^{4}$

Assim, o internato de MGF foca-se na capacitação de médicos para a prestação de cuidados abrangentes a todos os indivíduos que os procurem, permitindo 
adquirir competências nucleares para cuidar do doente no contexto da família, da comunidade e da cultura em que se insere, privilegiando uma abordagem holística, coordenada e continuada. ${ }^{3}$ Neste contexto, os quatro anos de formação específica em MGF assumem extrema importância na progressiva aquisição da capacidade de gestão da saúde dos utentes, bem como dos recursos existentes, garantindo a qualidade dos cuidados prestados.

Em dezembro de 2019, na cidade de Wuhan, surgiram os primeiros relatos de casos de uma doença infeciosa do sistema respiratório despoletada por um novo coronavírus, denominado severe acute respiratory syndrome coronavírus 2 (SARS-CoV-2). Posteriormente, a Organização Mundial da Saúde (OMS) anunciou oficialmente o nome desta patologia, que rapidamente se propagou pelo mundo inteiro: COVID-19.

Desde então assiste-se a uma emergente necessidade de reorganização da prestação dos cuidados de saúde, incluindo os CSP, de forma a dar resposta às exigências da pandemia. Neste âmbito, o internato de MGF vê-se revestido de profundas mudanças e eventuais condicionantes que merecem particular abordagem e cuidada reflexão.

\section{UM PERCURSO QUE SE DESVIA}

O programa nacional do internato de MGF visa a construção de um perfil profissional definido internacionalmente, ${ }^{5}$ assim como o desenvolvimento de competências nucleares que se incluem na definição europeia de MGF. ${ }^{3}$ Este prevê diferentes modalidades de desenvolvimento e concretiza-se mediante uma formação em estágio e uma formação teórico-prática. O eixo da sua estrutura é a formação em MGF, definindo-se a partir daí as áreas complementares, obrigatórias e opcionais. ${ }^{4}$

Neste sentido, no início do internato é elaborado um Plano Pessoal de Formação (PPF), ${ }^{4}$ que representa um instrumento de aprendizagem ao definir as opções formativas e a forma como estas acontecem no tempo. Enquanto o médico interno de MGF concretiza este plano, importa refletir sobre a plasticidade e a capacidade de encaixe com que o faz. De facto, a conjugação dos estágios em MGF, que constituem a base do PPF, com os estágios hospitalares, sejam estes obrigatórios ou facultativos, implica uma constante mudança de ro- tinas, adaptação a novas equipas e tentativa de integração. Se se associar a esta equação o fator pandemia por COVID-19 torna-se muito desafiador.

A partir de março de 2020, o PPF vê-se balançado na sua organização e estrutura, na medida em que o interno de MGF fica integrado em atividades até então não planeadas, mas de importância extrema face à situação emergente. Numa primeira fase da pandemia, e de acordo com as suas fases de formação, os internos passam a assumir diferentes tarefas, de que são exemplos a constituição das equipas das Áreas Dedicadas para Doentes Respiratórios (ADR), no caso dos internos dos últimos anos e a gestão da plataforma TRACE-COVID, consulta telefónica, pedidos de exames complementares de diagnóstico e renovação de receituário, no caso dos internos dos primeiros anos.

Por um lado, esta reformulação potenciou o desenvolvimento de competências inovadoras por parte dos internos dos últimos anos que integraram as ADR, valência até então desconhecida e, por isso mesmo, desafiante. Por outro lado, colocou os internos dos primeiros anos em tarefas mais isoladas e burocráticas, potencialmente menos estimulantes sob o ponto de vista clínico. Poder-se-á, assim, refletir sobre um possível desfasamento de realidades nos diferentes anos de internato, durante a primeira fase da pandemia, o que poderá ter repercussões em níveis que ainda se desconhecem, mas que poderão ser alvo de estudo.

No global fala-se de um percurso que se desvia para todos os anos de formação, sendo que pelo caminho se adiaram tarefas e opções formativas, sob prejuízo de não se virem a concretizar ou de retomar numa fase não tão oportuna. Fala-se em projetos curriculares que não iniciaram ou prosseguiram no tempo idealizado. Fala-se da tentativa de conjugação de novas funções com as tarefas curriculares, inclusive a preparação da avaliação final. E fala-se, novamente, em capacidade de adaptação e, sobretudo, em treino de resiliência durante este desvio.

Não obstante a clara importância que os internos de MGF, das mais diversas formas, representam no combate à pandemia, há, assim, um fio condutor que se quebra no exercício e aprendizagem da MGF.

\section{A RELAÇÃO MÉDICO-DOENTE}

Existe evidência científica robusta de que a relação 
médico-doente constitui um fator influenciador, ainda que em diferentes proporções, de alguns fenómenos, como os índices de satisfação dos doentes com a consulta médica e a adesão a cuidados promotores de saúde, sejam estes preventivos ou terapêuticos. ${ }^{6}$ Esta relação assume um papel bidirecional na medida em que interfere positivamente na identificação dos problemas de saúde e, de igual forma, contribui para a satisfação do médico em relação ao exercício da sua atividade profissional. ${ }^{7}$

Apesar de a sua importância ser transversal a todas as especialidades médicas, o MF ocupa um lugar privilegiado para o desenvolvimento de uma relação médico-doente estruturada e facilitadora. É neste sentido que o médico interno de MGF desde cedo procura desenvolver competências de entrevista clínica, adquirindo progressivamente estratégias de aperfeiçoamento da interação com o doente.

Se neste caminho o interno de MGF ainda teria algo a percorrer, juntam-se alguns passos a partir do momento em que fica privado do contacto direto e, numa primeira fase, da atividade assistencial programada. A pandemia por COVID-19 veio, de certo modo, acentuar a distância ao doente que, até então, o médico interno se tinha esforçado por encurtar. De facto, a utilização de equipamentos de proteção individual e mecanismos de verificação que o risco de infeção por SARS-CoV-2 impõe, aliado à necessidade de registos clínicos densos e demorados, que já constituía uma condicionante da consulta, poderão conjugar-se para o desvirtuar do treino da empatia que o internato ambiciona.

Por outro lado, o retomar da atividade assistencial traz ao médico interno a possibilidade de se reinventar na tentativa de recuperação desta distância, aproximando-se do doente na medida do necessário para compreender as suas novas fragilidades e necessidades e, assim, desenvolver novas competências.

Também dentro das novas competências se inclui o desenvolvimento da arte da consulta telefónica, cuja dimensão aumentou de forma incontornável no contexto atual. Existem limitações desta tipologia de consulta, como são os casos da impossibilidade de perceção da linguagem não-verbal ${ }^{8} \mathrm{e}$ de realização do exame físico que, para o interno, poderão representar condicionantes acrescidas, uma vez que não é portador de uma relação tão consolidada com o doente. Neste âm- bito, o interno terá de superar-se para ultrapassar estas limitações, bem como compreender os benefícios da utilização da consulta telefónica estruturada e bem aplicada, utilizando-a de forma mais eficiente e criteriosa em situações futuras.

Assim, ao interno de MGF, durante a pandemia por COVID-19, é colocado o desafio de prosseguir na aprendizagem da construção e consolidação da relação médico-doente, apesar das condicionantes já descritas, o que poderá encetar o desenvolvimento de competências indubitavelmente inovadoras. Acresce o facto de que consegue ter uma visão privilegiada da consulta telefónica, tanto como um complemento útil da consulta como uma ferramenta de literacia em saúde, na medida em que a evolução a este nível acontece no mesmo tempo da sua própria formação.

\section{O TRABALHO EM EQUIPA}

A OMS explica que, no âmbito das profissões relacionadas com a saúde, dever-se-ia dar a mesma importância às competências relacionais como às competências técnicas e cognitivas. ${ }^{9}$

A literatura reforça o mesmo pressuposto, sendo que os primeiros estudos sobre o tema concluíram que o trabalho em equipa proporciona não só ganhos em saúde como contribui para a satisfação do doente. ${ }^{10}$

No âmbito da MGF já seria indiscutível o valor do trabalho em equipa, pela multiplicidade e complexidade dos problemas de saúde com que os clínicos se confrontam, assim como pelo desenvolvimento tecnológico e científico que acrescem diariamente. Contudo, e apesar de conscientes da necessidade e dos frutos do trabalho interpares, a prática clínica diária em MGF foi-se pautando, ao longo dos anos, por alguma individualidade, sobretudo pela densidade e complexidade de utentes que constituem a lista de cada MF. Portanto, quer pelas vicissitudes do sistema quer pela multiplicidade de tarefas que a MGF se propõe a gerir, ou mesmo pelas características individuais de cada clínico, o trabalho em equipa poder-se-ia assumir como um conceito mais teórico do que vivido na prática. A pandemia por COVID-19 veio, inevitavelmente, conferir a este conceito o sentido prático que lhe faltava.

Desde o início que a pandemia impôs a reorganização das unidades de saúde, incluindo dos CSP, proporcionando-se, neste âmbito, condições particularmente 
provocadoras na aquisição de noções de trabalho em equipa e consciencialização da sua importância.

Numa primeira instância, ao fazer parte da linha da frente, o interno de MGF participou ativamente na criação de protocolos de atuação, fluxogramas e circuitos que visam a minimização do risco de contágio, bem como otimizam a abordagem ao doente com infeção por SARS-CoV-2. A este nível, o interno de MGF compreende que o trabalho articulado é uma imposição para se obter uma abordagem integrada, devendo existir, em troca, comunicação e cooperação em favor do doente, sendo que, para tal, o contexto deverá primar por ser orgânico e funcional. Também neste contexto o médico interno passa a assumir novas tarefas, particularmente a constituição das equipas de ADR que, para além de representar uma valência desconhecida, o descentraliza da sua área de conforto, colocando-o em contacto com entidades patológicas distintas e, por isso, encetando novas possibilidades de raciocínio clínico e de atuação.

Adicionalmente, ao implicar a reestruturação de equipas de trabalho no seio dos CSP, a pandemia por COVID-19 levou ao cruzar de elementos das diferentes áreas profissionais, incluindo médicos, enfermeiros, assistentes técnicos e operacionais das várias unidades de saúde. Neste âmbito, o interno de MGF encontra um espaço privilegiado para desenvolver competências comunicacionais ao ter de interagir com elementos e formas de trabalho distintas, o que certamente se revelará enriquecedor no seu percurso formativo e, no futuro, enquanto médico especialista.

Importa também referir que a literatura carece de estudos sobre o conhecimento que existe sobre o papel do médico interno de MGF, ou até acerca do que é o internato médico no geral, por parte dos vários profissionais das unidades de saúde. E, portanto, não menos interessante, acresce o facto de a pandemia constituir um holofote sobre o que é o internato médico no global, mas também sobre a capacidade de atuação e de polivalência do interno de MGF aos olhos da restante equipa. Este facto poderá contribuir para a validação do sentido de autonomia que o médico interno ambiciona, assim como para o crescimento, em diferentes vertentes, das equipas de saúde em que está inserido.

Assim, proporcionou-se o desenvolvimento de um conjunto de competências, motivadas pelas novas necessidades e exigências que a pandemia impôs, e que se traduzem em novas formas de trabalho, novas formas de equacionar o pensamento clínico e no reforço inequívoco do trabalho interpares e da comunicação eficaz como mais-valias. Ao ser aplicado na prática clínica futura, de forma transversal e incluindo a extensão a outras valências da prestação de cuidados, este novo conjunto de competências poder-se-á traduzir em ganhos em saúde potencialmente mensuráveis ao longo das próximas décadas.

\section{UMA ESCOLHA QUE BALANÇA OU CONSOLIDA?}

Nos últimos dez anos assistiu-se ao aumento da classificação média na prova nacional de seriação dos médicos que colocam em MGF a sua primeira escolha, contrariando a tendência dos anos anteriores. ${ }^{11-12}$ Apesar de a MGF se ter vindo a pautar por uma inquestionável evolução da sua vertente formativa e consequente reconhecimento, também a literatura carece de estudos que se debrucem sobre a avaliação da satisfação dos seus médicos internos.

Um estudo de 2014 revelou que os internos de MGF da Zona Norte se encontravam satisfeitos com o seu programa de formação específica, satisfação esta relacionada com fatores como a relação com o orientador de formação e o seu desempenho e a organização global do programa de formação. O mesmo estudo revelou que a eventual insatisfação se associava ao peso das atividades não clínicas para efeitos curriculares. ${ }^{13}$

Se se analisar o binómio interno-orientador revelase, para ambos, um fator preponderante na vivência da especialidade. A aprendizagem e o exercício da MGF executam-se, em diversos momentos, através da imitação. De forma mais ou menos consciente, o interno de MGF imita e adquire comportamentos do orientador de formação que se tornam intrínsecos à sua prática clínica, da mesma forma que o orientador progride com o exercício de tutoria. A pandemia por COVID- 19 veio tornar este binómio mais coeso e necessário, na medida em que a incerteza e a aquisição de conhecimentos numa área desconhecida acontecem para ambos em tempo real. Neste âmbito há uma relação que se estreita e que, por consequência, potenciará o crescimento mútuo em diferentes dimensões, que vão para além da pandemia.

Também durante uma primeira fase da pandemia, as tarefas curriculares não clínicas foram postas em 
segundo plano, em detrimento da atividade clínica, o que poderá ser um fator enriquecedor sob o ponto de vista da aquisição e consolidação de competências médicas e que parece ser algo que os internos ambicionam. Por outro lado, poderá representar um fator de ansiedade acrescida, sobretudo para os internos dos últimos anos, cuja tentativa de conciliação da fase final do internato com o combate à pandemia poderá ter-se revelado um exercício mais denso e exaustivo, sob o ponto de vista profissional e pessoal.

Assim sendo, compreende-se que a pandemia por COVID-19 poderá ter reforçado positivamente alguns fatores já conhecidos como potenciadores do grau da satisfação dos médicos internos com a especialidade, destacando-se a relação com o orientador de formação e a possibilidade acrescida de se dedicarem à componente clínica, diminuindo o tempo correspondente às restantes tarefas curriculares. Por outro lado, o facto de ter havido significativa entropia dentro da estruturação do plano de formação, assim como o acumular de tarefas curriculares que ficaram por cumprir, poderá ter influenciado negativamente a vivência do internato médico.

Se inicialmente foi dito que a literatura carece de estudos sobre os fatores influenciadores da satisfação durante o internato médico, a pandemia por COVID-19 veio, ao moldar variáveis já conhecidas e acrescentando novas, reafirmar esta necessidade. Propõe-se, assim, a época pandémica como um mote para aprofundar o conhecimento do internato médico em todas as suas vertentes, numa visão a 360 graus, e sobretudo explorando aquilo que constituem fragilidades e motivações dos médicos internos. Desta forma, poder-se-ão perspetivar reformulações, quer em termos formativos quer em termos de avaliação de desempenho, tão pertinentes quanto as que se assistiu desde a criação da especialidade.

\section{CONCLUSÃO}

Apesar de pautado pelos mesmos valores, humanistas e de vivência da profissão ao serviço do doente e da sociedade, o exercício médico durante e após a pandemia por COVID-19 sofreu mudanças inquestionáveis, que deverão encetar reflexões, tendo-se proposto neste artigo algumas que dizem respeito ao internato de MGF.
A pandemia por COVID-19 comprometeu o fio condutor da aprendizagem até então planeada da MGF ao implicar uma reformulação do plano de formação previamente delineado e, deste modo, arrastando tarefas e projetos curriculares que ficaram diluídos no tempo. Comprometeu o médico interno dos primeiros anos ao isolá-lo em tarefas menos estimulantes, sob o ponto de vista clínico, e comprometeu os internos dos anos mais avançados pela desafiante conciliação de múltiplas tarefas com a preparação da avaliação final. Comprometeu o treino e consolidação da aprendizagem da relação médico-doente que o médico interno ambiciona e que foi, de certo modo, interrompida.

Por outro lado, a pandemia por COVID-19 promete ao médico interno a aquisição de competências altamente inovadoras, sobretudo quando o coloca em áreas de atuação desconhecidas, particularizando a constituição das equipas de ADR, valência que o desafiou. Promete-lhe a compreensão de técnicas subjacentes à consulta telefónica, usando-a de forma criteriosa como um complemento útil da consulta e oferecendo-lhe mais um possível caminho para a literacia em saúde. Promete-lhe o enraizar do trabalho de equipa e o aperfeiçoamento da capacidade comunicacional, ferramentas fundamentais da sua prática clínica futura, mas que adicionalmente o tornaram mais visível e confiante na sua prática atual. Promete-lhe um lugar privilegiado de observação e estudo de um doente também ele diferente, moldado por novas necessidades e particularidades, e que se insurge durante o seu tempo de formação.

Posto isto, certamente muito mais é o que se promete do que o que ficou comprometido, sobretudo se for considerado que as promessas se poderão vir a traduzir em ganhos em saúde.

Eé neste sentido que se sugerem estudos focados na forma como estas novas competências poderão constituir elementos de valorização individual e curricular, equacionando-se, assim, reformulações pertinentes no âmbito do internato médico de medicina geral e familiar.

\section{REFERÊNCIAS BIBLIOGRÁFICAS}

1. Leeuwenhorst Working Party. The general practitioner in Europe [Internet]. Amsterdam: Leeuwenhorst; 1974. Available from: https://euract.woncaeurope.org/sites/euractdev/files/documents/archive/publi- 
cations/general-practitioner-europe-statement-working-party-appointed-2nd-european-conference-teaching.pdf

2. Haslam D. Introduction. In: The future of general practice: a statement by the Royal College of General Practitioners. London: Royal College of General Practitioners; 2014.

3. WONCA Europe. The European definition of general practice/family medicine [Internet]. WONCA Europe; 2011. Available from: https:// www.globalfamilydoctor.com/site/DefaultSite/filesystem/documents/regionDocs/European\%20Definition\%20of\%20general\%20pra ctice\%203rd\%20ed\%202011.pdf

4. Coordenação do Internato Complementar da Zona Norte. Caderneta de estágio 2021 [Internet]. Porto: CIMGFZN; 2021. Available from: http://www.arsnorte.min-saude.pt/wp-content/uploads/sites/3/2021/ 01/Caderneta_IM_2021.pdf

5. Heyrman J, editor. EURACT educational agenda of general practice/family medicine: tradução Portuguesa oficial da versão integral de 2005. Lisboa: Associação dos Docentes e Orientadores de Medicina Geral e Familiar; 2006.

6. Stewart MA, McWhinney IR, Buck CW. The doctor/patient relationship and its effect upon outcome. J R Coll Gen Pract. 1979;29(199):77-82.

7. Stewart MA. Effective physician-patient communication and health outcomes: a review. CMAJ. 1995;152(9):1423-33.

8. Van Galen LS, Car J. Telephone consultations. BMJ. 2018;360:k1047.

9. World Health Organization. Topic 4: being an effective team player [Internet]. Geneva:WHO; 2012. Available from: https://www.who.int/patientsafety/education/curriculum/who_mc_topic-4.pdf

10. Yodfat Y. A new method of teamwork in family medicine in Israel with the participation of nurses as physician's assistants. Am J Public Health. 1972;62(7):953-6.

11. Gaspar D. Escolher a especialidade de medicina geral e familiar: opção inicial ou uma alternativa? [The choice of family medicine as a specialty: the first choice or an alternative?]. Rev Port Clin Geral. 2010;26 (4):354-68. Portuguese

12. Gaspar D. Internato médico de medicina geral e familiar: quem somos? O perfil do médico interno de medicina geral e familiar em Portugal [General practice and family medicine vocational training: the specialty internship doctor's profile, in Portugal]. Acta Med Port. 2010;23(1):3950. Portuguese

13. Azevedo A, Domingues B, Moura J, Santos L. Estão os médicos internos satisfeitos com o internato de medicina geral e familiar? [Are family medicine trainees satisfied with their training program?]. Rev Port Med Geral Fam. 2014;30(1):24-30. Portuguese

\section{CONFLITO DE INTERESSES}

As autoras declaram não possuir quaisquer conflitos de interesse.

\section{ENDEREÇO PARA CORRESPONDÊNCIA}

Ana Margarida Dias Marques

E-mail: anamargarida.dm@gmail.com

https://orcid.org/0000-0001-6287-190X

Recebido em 14-02-2021

Aceite para publicação em 02-05-2021

\begin{abstract}
FAMILY MEDICINE INTERNSHIP IN A PANDEMIC: WHAT (COM)PROMISES?

The family medicine internship curriculum corresponds to a well-defined continuous medical training that in March 2020 was impaired in its structure due to the mobilization of intern doctors for unplanned areas of activity, but extremely important to combat the COVID-19 pandemic. In this context, there was a departure from the training curriculum, which although felt differently in different years of the internship, proved to be transversal to all, with a potential break in the guiding thread in the learning of family medicine. Nevertheless, the pandemic has become a driving force for the intern doctor to develop new skills, in areas to be explored, like the development of teamwork and communication skills, as well as the improvement of telephone consultation techniques. The internal-guiding binomial also became more cohesive, through joint learning in real-time, and there was a focus on clinical activity, to the detriment of curricular tasks. We suggest studies that continue to focus on the degree of motivation of intern physicians of family medicine, and how their new skills may constitute elements of individual valorization.
\end{abstract}

Keywords: COVID-19; Pandemic; Medical internship; Family medicine. 